This is the accepted manuscript made available via CHORUS. The article has been published as:

\title{
Hyperon-Nucleon Interactions from Quantum Chromodynamics and the Composition of Dense Nuclear Matter
}

S. R. Beane, E. Chang, S. D. Cohen, W. Detmold, H.-W. Lin, T. C. Luu, K. Orginos, A. Parreño, M. J. Savage, and A. Walker-Loud (NPLQCD Collaboration) Phys. Rev. Lett. 109, 172001 - Published 23 October 2012 DOI: 10.1103/PhysRevLett.109.172001 


\title{
Hyperon-Nucleon Interactions from Quantum Chromodynamics and the Composition of Dense Nuclear Matter
}

\author{
S.R. Beane, ${ }^{1}$ E. Chang, ${ }^{2}$ S.D. Cohen, ${ }^{3}$ W. Detmold,${ }^{4,5}$ H.-W. Lin, ${ }^{3}$ \\ T.C. Luu, ${ }^{6}$ K. Orginos, ${ }^{4,5}$ A. Parreño, ${ }^{2}$ M.J. Savage, ${ }^{3}$ and A. Walker-Loud ${ }^{7,8}$ \\ (NPLQCD Collaboration) \\ ${ }^{1}$ Department of Physics, University of New Hampshire, Durham, NH 03824-3568, USA \\ ${ }^{2}$ Dept. d'Estructura i Constituents de la Matèria. Institut de Ciències del Cosmos (ICC), \\ Universitat de Barcelona, Martí Franquès 1, E08028-Spain \\ ${ }^{3}$ Department of Physics, University of Washington, Seattle, WA 98195-1560. \\ ${ }^{4}$ Department of Physics, College of William and Mary, Williamsburg, VA 23187-8795, USA \\ ${ }^{5}$ Jefferson Laboratory, 12000 Jefferson Avenue, Newport News, VA 23606, USA \\ ${ }^{6} N$ Division, Lawrence Livermore National Laboratory, Livermore, CA 94551, USA \\ ${ }^{7}$ Lawrence Berkeley National Laboratory, Berkeley, CA 94720, USA \\ ${ }^{8}$ Department of Physics, University of California, Berkeley, CA 94720, USA
}

(Dated: September 11, 2012)

\begin{abstract}
The low-energy $n \Sigma^{-}$interactions determine, in part, the role of the strange quark in dense matter, such as that found in astrophysical environments. The scattering phase shifts for this system are obtained from a numerical evaluation of the QCD path integral using the technique of lattice QCD. Our calculations, performed at a pion mass of $m_{\pi} \sim 389 \mathrm{MeV}$ in two large lattice volumes, and at one lattice spacing, are extrapolated to the physical pion mass using effective field theory. The interactions determined from lattice QCD are consistent with those extracted from hyperon-nucleon experimental data within uncertainties, and strengthen model-dependent theoretical arguments that the strange quark is a crucial component of dense nuclear matter.
\end{abstract}

The interactions between hyperons and nucleons are important for understanding the composition of dense nuclear matter. In high-density baryonic systems, the large values of the Fermi energies may make it energetically advantageous for some of the nucleons to transform into hyperons via the weak interactions, with the increase in rest mass being more than compensated for by the decrease in combined Fermi energy of the baryon-lepton system. This is speculated to occur in the interior of neutron stars, but a quantitative understanding of this phenomenon depends on knowledge of the hyperon-nucleon $(\mathrm{YN})$ interactions in the medium. In this letter we use $n \Sigma^{-}$scattering phase shifts in the ${ }^{1} S_{0}$ and ${ }^{3} S_{1}$ spinchannels calculated with Lattice QCD (LQCD) to quantify the energy shift of the $\Sigma^{-}$hyperon in dense neutron matter, as might occur in the interior of a neutron star. Our results strongly suggest an important role for strangeness in such environments.

Precise nucleon-nucleon $(\mathrm{NN})$ interactions constrained by experiment and chiral symmetry, together with numerically small but important three-nucleon interactions, have served as input to refined many-body techniques for studying the structure of nuclei, such as Green-function Monte-Carlo [1], the No-Core Shell Model [2], or lattice effective field theory [3], which have led to remarkably successful calculations of the ground states and excited states of light nuclei, with atomic number $A<14$. By contrast, the YN potentials, which are essential for a firstprinciples understanding of the hypernuclei and dense matter, are only very-approximately known. Therefore, gaining a quantitative understanding of $\mathrm{YN}$ interactions
- on a par with knowledge of the $\mathrm{NN}$ interactions through experimental and LQCD methods, is a fundamental goal of nuclear science.

Existing experimental information about the YN interaction comes from the study of hypernuclei $[4,5]$, the analysis of associated $\Lambda$-kaon and $\Sigma$-kaon production in NN collisions near threshold [6-11], hadronic atoms [12], and from charge-exchange production of hyperons in emulsions and pixelated scintillation devices [13]. There are only a small set of cross-section measurements of the YN processes, and not surprisingly, the extracted scattering parameters are not accurately known. The potentials developed by the Nijmegen [14-16] and Jülich [1719] groups are just two examples of phenomenological models based on meson exchange, but the couplings in such models are not completely determined by the NN interaction and are instead obtained by a fit to the available YN data. In Refs. [14, 15], for example, six different models are constructed, each describing the available YN cross-section data equally well, but predicting different values for the phase shifts. Effective field theory (EFT) descriptions have also been developed [20-24] and have the advantage of being model independent.

In the absence of precise experimental measurements, LQCD calculations can be used to constrain the YN interactions. Several years ago, the NPLQCD Collaboration performed the first $n_{f}=2+1$ LQCD calculations of YN interactions [25] (and NN interactions [26]) at unphysical pion masses. Quenched and dynamical calculations were subsequently performed by the HALQCD Collaboration [27] and by NPLQCD [28]. Recent work by 
NPLQCD [29-31] and HALQCD [32, 33] has shown that the $S=-2 \mathrm{H}$-dibaryon is bound for pion masses larger than those of nature, and NPLQCD [31] has shown that the same is true for the $\Xi^{-} \Xi^{-}$with $S=-4$. In this letter, we use the results of LQCD calculations to determine leading-order (LO) couplings of the YN EFT (using Weinberg power counting [24]) which in turn allow for a determination of $\mathrm{YN}$ interactions at the physical pion mass.

In LQCD, Lüscher's method [34-37] can be employed to extract two-particle scattering amplitudes below inelastic thresholds. For a single scattering channel, the deviation of the energy eigenvalues of the two-hadron system in the lattice volume from the sum of the singlehadron masses is related to the scattering phase shift $\delta(q)$. The Euclidean time behavior of LQCD correlation functions of the form $C_{\chi}(t)=\left\langle 0\left|\chi(t) \chi^{\dagger}(0)\right| 0\right\rangle$, where $\chi$ represents an interpolating operator with the quantum numbers of the one-particle or two-particle systems under consideration, determines the ground-state energies of the one-particle and two-particle systems, $E_{1}^{A, B}=m_{A, B}$ and $E_{2}^{(A B)}=\sqrt{q^{2}+m_{A}^{2}}+\sqrt{q^{2}+m_{B}^{2}}$, respectively. The form of the interpolating operators and the methodology used for extracting the energy shift are discussed in detail in Ref. [38]. By computing the masses of the particles and the ground-state energy of the two-particle system, one obtains the squared momentum $q^{2}$, which can be either positive or negative. For s-wave scattering below inelastic thresholds, $q^{2}$ is related to the real part of the inverse scattering amplitude through the eigenvalue equation [35] (neglecting phase shifts in $l \geq 4$ partial-waves):

$$
q \cot \delta(q)=\frac{1}{\pi L} \lim _{\Lambda \rightarrow \infty} \sum_{\mathbf{j}}^{|\mathbf{j}|<\Lambda} \frac{1}{|\mathbf{j}|^{2}-q^{2}\left(\frac{L}{2 \pi}\right)^{2}}-4 \pi \Lambda .
$$

This relation enables a LQCD determination of the value of the phase shift at the momentum $\sqrt{q^{2}}$.

Determining the ground-state energy of a system in multiple lattice volumes allows for bound states to be distinguished from scattering states. A bound state corresponds to a pole in the $\mathrm{S}$ matrix, and in the case of a single scattering channel, is signaled by $\cot \delta(q) \rightarrow+i$ in the large-volume limit. With calculations in two or more lattice volumes that both have $q^{2}<0$ and $q \cot \delta(q)<0$ it is possible using Eq. (1) to perform an extrapolation to infinite volume to determine the binding energy of the bound state $B_{\infty}=\gamma^{2} / m$, where $\gamma$ is the binding momentum [35-37]. The range of nuclear interactions is determined by the pion mass, and therefore the use of Lüscher's method requires that $m_{\pi} L \gg 1$ to strongly suppress the contributions that depend exponentially upon the volume, $e^{-m_{\pi} L}[39]$. However, corrections of the form $e^{-\gamma L}$, where $\gamma^{-1}$ is approximately the size of the bound state, must also be small for the infinite volume extrapolation to rapidly converge.

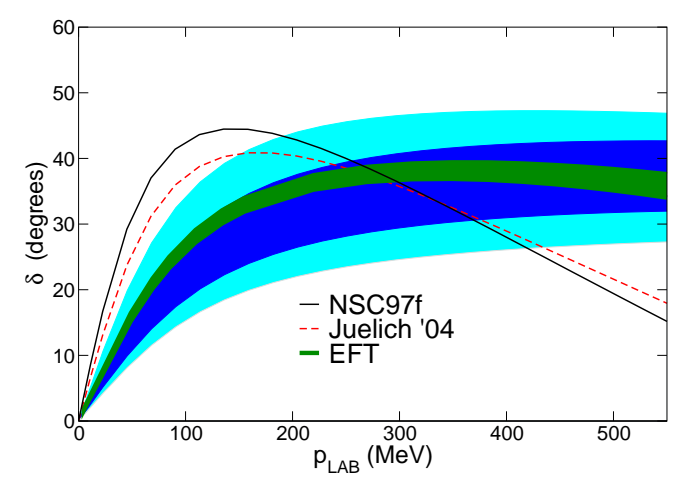

FIG. 1: LQCD-predicted ${ }^{1} S_{0} n \Sigma^{-}$phase shift versus laboratory momentum at the physical pion mass (blue bands), compared with other determinations, as discussed in the text.

Our results are from calculations on two ensembles of $n_{f}=2+1$ anisotropic clover gauge-field configurations $[40,41]$ at a pion mass of $m_{\pi} \sim 389 \mathrm{MeV}$, a spatial lattice spacing of $b_{s} \sim 0.123(1) \mathrm{fm}$, an anisotropy of $\xi=b_{s} / b_{t} \sim 3.5$, and with spatial extents of 24 and 32 lattice sites, corresponding to spatial dimensions of $L \sim 3.0$ and $3.9 \mathrm{fm}$, respectively, and temporal extents of 128 , and 256 lattice sites, respectively. A detailed analysis demonstrates that the single-baryon masses in these lattice ensembles are effectively in the infinite-volume limit [42], and that exponential volume corrections can be neglected in this work. Lüscher's method assumes that the continuum single-hadron energy-momentum relation is satisfied over the range of energies used in the eigenvalue equation in Eq. (1). As discussed in Refs. [29, 31], the uncertainties in the energy-momentum relation translate to a $2 \%$ uncertainty in the determination of $q^{2}$.

We focus on ${ }^{1} S_{0}$ and ${ }^{3} S_{1} n \Sigma^{-}$interactions, $N \Sigma$ in the $I=3 / 2$ channel, and do not consider the $I=1 / 2 N \Sigma$ $N \Lambda$ coupled channels. Calculations in the $I=1 / 2$ channel are complicated by the proximity in energy of the ground and first-excited levels in the finite volume. Moreover, while the $\Sigma$ is more massive than the $\Lambda$, the presence of $\Lambda$ 's in dense matter does not lower the electron Fermi energy. In the limit of $\mathrm{SU}(3)$ flavor symmetry, the ${ }^{1} S_{0}$-channels are in symmetric irreducible representations of $\mathbf{8} \otimes \mathbf{8}=\mathbf{2 7} \oplus \mathbf{1 0} \oplus \overline{\mathbf{1 0}} \oplus \mathbf{8} \oplus \mathbf{8} \oplus \mathbf{1}$, and hence the $n \Sigma^{-}$(along with the $\Xi^{-} \Xi^{-}, \Sigma^{-} \Sigma^{-}, n n$, and $\Sigma^{-} \Xi^{-}$) transforms in the 27. YN and NN scattering data along with the leading $\mathrm{SU}(3)$ breaking effects, arising from the light-meson and baryon masses, suggest that all of these channels are attractive at the physical pion mass, and that $\Xi^{-} \Xi^{-}$and $\Sigma^{-} \Sigma^{-}$are bound [43-45]. By contrast, the ${ }^{3} S_{1}$-channel of $n \Sigma^{-}$scattering transforms in the $\mathbf{1 0}$ in the limit of SU(3) symmetry, and is therefore unrelated to NN interactions. Hence, this channel is quite uncertain, with disagreements among hadronic models as to whether the interaction is attractive or repulsive.

The low-energy $n \Sigma^{-}$interactions can be described by 


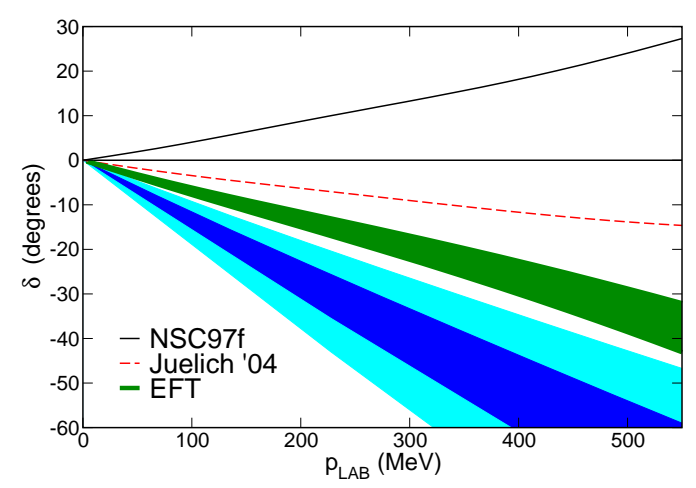

FIG. 2: LQCD-predicted ${ }^{3} S_{1} n \Sigma^{-}$phase shift versus laboratory momentum at the physical pion mass (blue bands), compared with other determinations, as discussed in the text.

an EFT of nucleons, hyperons and pseudoscalar mesons $(\pi, \mathrm{K}$ and $\eta)$, constrained by chiral symmetry $[20,23,24]$. At leading order (LO) in the expansion, the $n \Sigma^{-}$interaction is given by one-meson exchange together with a contact operator that encodes the low-energy effect of short-distance interactions. As these contact operators are independent of the light-quark masses, at LO the quark-mass dependence of the $n \Sigma^{-}$interactions is dictated by the meson masses. Therefore, in each partial wave, a single lattice datum at a sufficiently low pion mass determines the coefficient of the contact operator, thereby determining the LO interaction, including energy-independent and local potentials, wavefunctions and phase shifts, at the physical pion mass.

We find that our LQCD calculations in the ${ }^{1} S_{0} n \Sigma^{-}$ channel are consistent with the $\mathrm{SU}(3)$ symmetry expectations. At $m_{\pi} \sim 389 \mathrm{MeV}$, using a volume extrapolation as discussed above, we find that this channel has a bound state, with binding energy $B=25 \pm 9.3 \pm 11 \mathrm{MeV}$. The quality of the LQCD data in the ${ }^{1} S_{0} n \Sigma^{-}$channel is comparable to that of its 27-plet partner $\Xi^{-} \Xi^{-}$, analyzed in detail in Ref. [31] (see also [46]). In the EFT, the coefficient of the LO contact operator in this channel is determined by tuning it to reproduce the LQCD-determined binding energy. We find that this channel becomes unbound at $m_{\pi} \lesssim 300 \mathrm{MeV}$, in agreement with Ref. [47], which constrained the LO contact operator using experimental data. In Fig. 1, we show the predicted ${ }^{1} S_{0} n \Sigma^{-}$ phase shift at the physical pion mass - (dark, light) blue bands correspond to (statistical, systematic) uncertainties - and compare with the EFT constrained by experimental data [24], the Nijmegen NSC97f model [14], and the Jülich '04 model [19]. The systematic uncertainties on our predictions include those arising from the LQCD calculation (see [46]) as well as estimates of omitted higher-order effects in the EFT.

The ${ }^{3} S_{1}{ }^{3} D_{1} n \Sigma^{-}$coupled channel is found to be highly repulsive in the s-wave at $m_{\pi} \sim 389 \mathrm{MeV}$, requiring interactions with a hard repulsive core of extended size.
Such a core, if large enough, would violate a condition required to use Lüscher's relation, namely $R \ll L / 2$ where $R$ is the range of the interaction. We have determined the EFT potential directly by solving the 3-dimensional Schrödinger equation in finite volume to reproduce the energy levels obtained in the LQCD calculations. The repulsive core, whose size is set by the contact operator at LO in the EFT, is found to be strongly enhanced over the meson-exchange contributions. This formally precludes the use of Lüscher's relation, but both methods lead to phase shifts that agree within uncertainties, indicating that the exponential corrections to Lüscher's relation are small. In Fig. 2, we show the predicted ${ }^{3} S_{1}$ $n \Sigma^{-}$phase shift at the physical pion mass.

The $n \Sigma^{-}$interactions presented here are the crucial ingredient in calculations that address whether $\Sigma^{-}$'s appear in dense neutron matter. As a first step, and in order to understand the competition between attractive and repulsive components of the $n \Sigma^{-}$interaction, we adopt a result due to Fumi for the energy shift due to a static impurity in a non-interacting Fermi system [48]:

$$
\Delta E=-\frac{1}{\pi \mu} \int_{0}^{k_{f}} d k k\left[\frac{3}{2} \delta_{{ }_{3} S_{1}}(k)+\frac{1}{2} \delta_{1 S_{0}}(k)\right],
$$

where $\mu$ is the reduced mass in the $n \Sigma^{-}$system. Using our LQCD determinations of the phase shifts, and allowing for a $30 \%$ theoretical uncertainty, the resulting energy shift and uncertainty band is shown in Fig. 3. At neutron number density $\rho_{n} \sim 0.4 \mathrm{fm}^{-3}$, which may be found in the interior of neutron stars, the neutron chemical potential is $\mu_{n} \sim M_{N}+150 \mathrm{MeV}$ due to neutronneutron interactions, and the electron chemical potential, $\mu_{e^{-}} \sim 200 \mathrm{MeV}$ [49]. Therefore $\mu_{n}+\mu_{e^{-}} \sim 1290 \mathrm{MeV}$, and consequently, if $\mu_{\Sigma^{-}}=M_{\Sigma}+\Delta E \lesssim 1290 \mathrm{MeV}$, that is, $\Delta E \lesssim 100 \mathrm{MeV}$, then the $\Sigma^{-}$, and hence the strange quark, will play a role in the dense medium. We find using Fumi's theorem that $\Delta E=46 \pm 13 \pm 24 \mathrm{MeV}$ at $\rho_{n}=0.4 \mathrm{fm}^{-3}$. Corrections due to correlations among neutrons are difficult to estimate and will require manybody calculations which are beyond the scope of this study. Despite this caveat, the results shown in Fig. 3 indicate that the repulsion in the $n \Sigma^{-}$system is inadequate to exclude the presence of $\Sigma^{-}$'s in neutron star matter, a conclusion that is consistent with most of the previous phenomenological modeling (for a recent review, see Ref. [50]). The present uncertainty in the hadronic interactions, and their transcription into many-body calculations of dense matter, have led to a large uncertainty in the composition of dense matter, particularly the precise role of the strange quark (see for instance Refs. [51] and [52]). Our present calculations provide a step towards eliminating the uncertainties arising from the interactions among hadrons.

In this letter, we have presented the first LQCD predictions for hypernuclear physics, the ${ }^{1} S_{0}$ and ${ }^{3} S_{1} n \Sigma^{-}$scattering phase shifts shown in Fig. 1 and Fig. 2. While the 


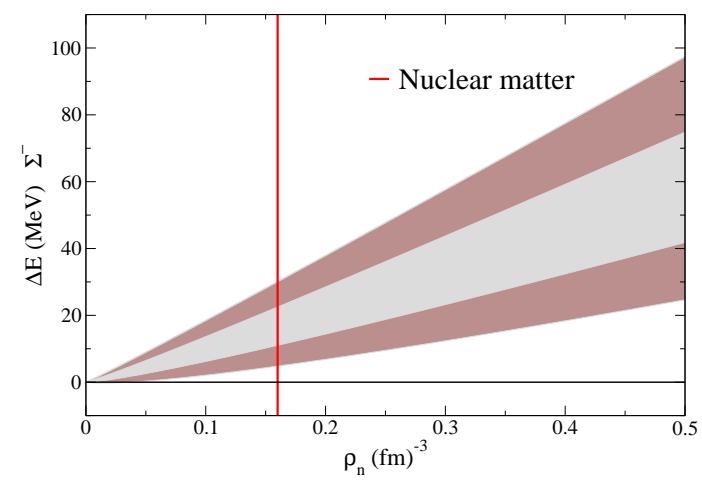

FIG. 3: The energy shift versus neutron density of a single $\Sigma^{-}$in a non-interacting Fermi gas of neutrons as determined from Fumi's theorem in Eq. (2). The inner (outer) band encompasses statistical (systematic) uncertainties.

LQCD calculations have been performed at a single lattice spacing, lattice-spacing artifacts are expected to be smaller than the other systematic uncertainties. We anticipate systematically refining the analysis presented in this letter as greater computing resources become available. The $n \Sigma^{-}$interaction is critical in determining the relevance of hyperons in dense neutron matter, and we have used the LQCD predictions of the phase shifts to estimate the $\Sigma^{-}$energy shift in the medium. Our calculation suggests that hyperons are important degrees of freedom in dense matter, consistent with expectations based upon the available experimental data and hadronic modeling. It is important that more sophisticated many-body techniques be combined with the interactions determined in this work to obtain a more precise determination of the energy shift of the $\Sigma^{-}$in medium. This will refine the prediction for the role of strange quarks in astrophysical environments, and, in particular, will quantitatively address questions posed by the recent observation of a $1.9 M_{\odot}$ neutron star [53].

We thank J. Haidenbauer, S. Reddy, K. Roche and A. Torok for valuable conversations, and R. Edwards and B. Joó for help with QDP++ and Chroma [54]. We acknowledge computational support from the USQCD SciDAC project, NERSC and ALCF (Office of Science of the DOE, DE-AC02-05CH11231 and DE-AC02-06CH11357), the UW Hyak facility (NSF PHY-09227700), BSCCNS (Barcelona), LLNL, and XSEDE, which is supported by NSF grant OCI-1053575. We acknowledge support by the NSF grants CAREER PHY-0645570, PHY-0555234, and CCF-0728915; by DOE grants DE-FG03-97ER4014, DE-AC05-06OR23177, DE-FG0204ER41302, OJI DE-SC0001784, DE-FC02-06ER41443, DE-AC02-05CH11231; by FIS2008-01661 from MEC and FEDER; by Jeffress Memorial Trust grant J-968.
[1] S. C. Pieper, Riv. Nuovo Cim. 31, 709 (2008).

[2] P. Navratil, S. Quaglioni, I. Stetcu and B. R. Barrett, J. Phys. G G 36, 083101 (2009).

[3] E. Epelbaum, H. Krebs, D. Lee and U. -G. Meissner, Phys. Rev. Lett. 104, 142501 (2010).

[4] A. Gal, E. Hungerford, Nucl. Phys. A 754 1-489 (2005).

[5] O. Hashimoto and H. Tamura, Prog. Part. Nucl. Phys. 57, 564 (2006).

[6] J. Balewski et al., Phys. Lett. B 420, 211 (1998).

[7] S. Sewerin et al., Phys. Rev. Lett. 83, 682 (1999).

[8] P. Kowina et al., Eur. Phys. j. A22, 293 (2004).

[9] R. Bilger et al., Phys. Lett. B 420, 217 (1998).

[10] M. Abdel-Bary et al., Phys. Lett. B 595, 127 (2004).

[11] A. Gasparyan, J. Haidenbauer, C. Hanhart, J. Speth, Phys. Rev. C 69, 034006 (2004).

[12] C. J. Batty, E. Friedman and A. Gal, Phys. Rept. 287 (1997) 385.

[13] J. K. Ahn et al. [KEK-PS E289 Collaboration], Nucl. Phys. A 761, 41 (2005).

[14] Th.A. Rijken, V.G.J. Stoks and Y. Yamamoto, Phys. Rev. C 59, 21 (1999).

[15] Th.A. Rijken, Y. Yamamoto, Phys. Rev. C 73044008 (2006).

[16] T. A. Rijken, M. M. Nagels and Y. Yamamoto, Prog. Theor. Phys. Suppl. 185, 14 (2010).

[17] B. Holzenkamp, K. Holinde, and J. Speth, Nucl. Phys. A 500 (1989) 485.

[18] A. Reuber, K. Holinde, H.-C. Kim, and J. Speth, Nucl. Phys. A 608243 (1996).

[19] J. Haidenbauer and U.-G. Meißner, Phys. Rev. C 72 044005 (2005).

[20] M. J. Savage and M. B. Wise, Phys. Rev. D 53, 349 (1996).

[21] C.L. Korpa, A.E.L. Dieperink, and R.G.E. Timmermans, Phys. Rev. C 65, 015208 (2001).

[22] H.W. Hammer, Nucl. Phys. A 705, 173 (2002).

[23] S.R. Beane, P.F. Bedaque, A. Parreño, M.J. Savage, Nucl. Phys. A 747, 55 (2005).

[24] H. Polinder, J. Haidenbauer and Ulf-G. Meißner, Nucl. Phys. A 779, 244 (2006) [arXiv:nucl-th/0605050].

[25] S. R. Beane et al., [NPLQCD], Nucl. Phys. A 794, 62 (2007).

[26] S. R. Beane, P. F. Bedaque, K. Orginos and M. J. Savage, Phys. Rev. Lett. 97, 012001 (2006) [hep-lat/0602010].

[27] H. Nemura, et al., Phys. Lett. B 673, 136 (2009).

[28] S. R. Beane et al., [NPLQCD Collaboration], Phys. Rev. D 81, 054505 (2010).

[29] S. R. Beane et al., [NPLQCD Collaboration], Phys. Rev. Lett. 106, 162001 (2011) [arXiv:1012.3812 [hep-lat]].

[30] S. R. Beane et al., Mod. Phys. Lett. A 26, 2587 (2011) [arXiv:1103.2821 [hep-lat]].

[31] S. R. Beane et al. [NPLQCD Collaboration], Phys. Rev. D in press, 2012 [arXiv:1109.2889 [hep-lat]].

[32] T. Inoue et al. [HAL QCD Collaboration], Phys. Rev. Lett. 106, 162002 (2011) [arXiv:1012.5928 [hep-lat]].

[33] T. Inoue et al. [HAL QCD Collaboration], arXiv:1112.5926 [hep-lat].

[34] H. W. Hamber, et al., Nucl. Phys. B 225, 475 (1983).

[35] M. Lüscher, Commun. Math. Phys. 105, 153 (1986).

[36] M. Lüscher, Nucl. Phys. B 354, 531 (1991).

[37] S. R. Beane et al., [NPLQCD], Phys. Lett. B 585, 106 
(2004).

[38] S. R. Beane et al., Prog. Part. Nucl. Phys. 66, 1, (2011).

[39] I. Sato and P. F. Bedaque, Phys. Rev. D 76, 034502 (2007).

[40] H. W. Lin et al. [HS], Phys. Rev. D 79, 034502 (2009).

[41] R. G. Edwards, B. Joo and H. W. Lin, Phys. Rev. D 78, 054501 (2008).

[42] S. R. Beane et al., [NPLQCD], Phys. Rev. D 84, 014507 (2011) [Phys. Rev. D 84, 039903 (2011)].

[43] V. G. J. Stoks and T. A. Rijken, Phys. Rev. C 59, 3009 (1999) [arXiv:nucl-th/9901028].

[44] G. A. Miller, arXiv:nucl-th/0607006.

[45] J. Haidenbauer, U.-G. Meißner, Phys. Lett. B684, 275280 (2010). [arXiv:0907.1395 [nucl-th]].

[46] S. R. Beane et al., [NPLQCD], in preparation.

[47] J. Haidenbauer and U.-G. Meissner, arXiv:1111.4069 [nucl-th] to appear in Nucl. Phys. A.

[48] G.D. Mahan, Many-Particle Physics, Plenum Press, NY
(1981).

[49] M. Baldo, G. F. Burgio and H. J. Schulze, Phys. Rev. C 61, 055801 (2000) [nucl-th/9912066].

[50] J. Schaffner-Bielich, Nucl. Phys. A 835, 279 (2010) [arXiv:1002.1658 [nucl-th]].

[51] S. Weissenborn, D. Chatterjee and J. SchaffnerBielich, Nucl. Phys. A 881, 62 (2012) [arXiv:1111.6049 [astro-ph.HE]]; Phys. Rev. C 85, 065802 (2012) [arXiv:1112.0234 [astro-ph.HE]].

[52] D. L. Whittenbury, J. D. Carroll, A. W. Thomas, K. Tsushima and J. R. Stone, arXiv:1204.2614 [nucl-th].

[53] P. Demorest, T. Pennucci, S. Ransom, M. Roberts and J. Hessels, Nature 467, 1081 (2010) [arXiv:1010.5788 [astro-ph.HE]].

[54] R. G. Edwards and B. Joo, Nucl. Phys. Proc. Suppl. 140 (2005) 832 . 